\title{
Synthesis of Colloidal ZnO Nanoparticles and Deposit of Thin Films by Spin Coating Technique
}

\author{
Jose Alberto Alvarado, ${ }^{1}$ A. Maldonado, ${ }^{2}$ H. Juarez, ${ }^{3}$ and M. Pacio ${ }^{3}$ \\ ${ }^{1}$ Centro de Investigaciones y de Estudios Avanzados del Instituto Politecnico Nacional, Programa de Nanociencias y Nanotecnología, \\ Avenida Instituto Politécnico Nacional No. 2508, Col. San Pedro Zacatenco, C.P. 07360, Mexico \\ ${ }^{2}$ Centro de Investigaciones y de Estudios Avanzados del Instituto Politecnico Nacional (CINVESTAV-IPN), Seccion de Electronica del \\ Estado Solido, Avenida Instituto Politécnico Nacional No. 2508, Col. San Pedro Zacatenco, C.P. 07360, Mexico \\ ${ }^{3}$ Centro de Investigaciones en Dispositivos Semiconductores, Benemérita Universidad Autónoma de Puebla, \\ Ciudad Universitaria Avenida San Claudio y 14 Sur, 72570 Puebla, Mexico
}

Correspondence should be addressed to Jose Alberto Alvarado; jalvarado@cinvestav.mx

Received 16 July 2013; Accepted 9 October 2013

Academic Editor: Yanbao Zhao

Copyright (C) 2013 Jose Alberto Alvarado et al. This is an open access article distributed under the Creative Commons Attribution License, which permits unrestricted use, distribution, and reproduction in any medium, provided the original work is properly cited.

$\mathrm{ZnO}$ colloidal nanoparticles were synthesized, the average size of these nanoparticles is around $25 \mathrm{~nm}$ with hexagonal form. It was noted that stabilization depends directly on the purifying process; in this work we do not change the nature of the solution as a difference from Meulekamp's method, and we do not use any alkanes to remove the byproducts; only a centrifuge to remove those ones was used, thereby the stabilization increases up to 24 days. It is observed from the results that only three times of washing is enough to prevent the rapid aging process. The effect of annealing process on the composition, size, and geometrical shape of $\mathrm{ZnO}$ nanoparticles was studied in order to know whether the annealing process affects the crystallization and growth of the nanoparticles. After the synthesis, the colloidal nanoparticles were deposited by spin coating technique showing that the formed nanoparticles have no uniformly deposition pattern. But is possible to deposit those ones in glass substrates. A possible deposition process of the nanoparticles is proposed.

\section{Introduction}

The study of semiconductor nanoparticles has been done from the last decades. These materials have been used for a lot of application areas; in particular casezinc oxide is an interesting material because of its wide range of applications for electronic [1], optic [2], and piezoelectric devices [3]. The development of nanoscience and nanotechnology research on ceramic materials shows a lot of promising applications of $\mathrm{ZnO}$ in the manufacturing of nanoscale-based electronic and optoelectronic devices, because of its abundance, nontoxicity, and the fact that it is a wide band gap semiconductor with good carrier mobility. Furthermore, it can also be doped both n-type and p-type [4-7].

Regarding the different applications of $\mathrm{ZnO}$ at nanometric scale, there has been interest in the development of synthesizing methods to obtain well-dispersed $\mathrm{ZnO}$ particles with controlled size and shape. $\mathrm{ZnO}$ nanoparticles have been deposited from physical [8] and chemical techniques. In the deposition of $\mathrm{ZnO}$ nanoparticles, chemical techniques are cheap and safe and can be implemented easily in a standard laboratory, as it was used in this work. $\mathrm{ZnO}$ nanoparticles with a size, shape, and purity matching well with those obtained in this project are compared with those based on physical techniques. The approach of these required characteristics (size and shape) has also been experienced in microwave [9], hydrothermal [10, 11], microemulsion [12], and sol-gel techniques.

Solution method is one of the cheapest techniques for the manufacturing of $\mathrm{ZnO}$ nanoparticles that have been reported recently with controlled characteristics. It is based on the liquid chemical reaction of zinc acetate and some compounds, containing $\mathrm{OH}$, such as sodium hydroxide $[13,14]$. In some cases, the growth of nanostructures demands high temperature, complex reactions, and even sophisticated equipment. However, this simple solution method has been 
reported to yield $\mathrm{ZnO}$ nanosized particles [15]. The role of the starting reactants, their concentrations, the reaction temperature, and time of the presence on dispersants has also been established in the formation of $\mathrm{ZnO}$ particles [16]. In addition, the time and the way that reagents are mixed also affect the nucleation and crystal growth [17]. The aging of the nanoparticles is one of the problems that researchers are trying to stop and develop the time that could be maintained stables in the medium; if they start to agglomerate, a big problem is present in the deposition time, and homogeneous thin films could not be obtained.

This work presents the study of the effect of the washing process as a determinant factor of $\mathrm{ZnO}$ nanoparticles stabilization, obtained from solution method, starting with zinc acetate and $\mathrm{KOH}$. The structure and grain size of $\mathrm{ZnO}$ nanoparticles, as a function of the washing steps, were analyzed with X-ray diffraction. Transmission electron microscopy (TEM) shows particles with hexagonal shape, with a narrow distribution of the crystallite size. From the transmittance spectrum obtained with Fourier transform infrared spectroscopy (FTIR), the chemical composition of the colloidal $\mathrm{ZnO}$ nanoparticles was analyzed, clearly showing the characteristic vibrational modes of $\mathrm{ZnO}$ that are around $430 \mathrm{~cm}^{-1}$.

Also these nanoparticles were deposited on glass substrates by spin coating process that is useful to deposit the formed nanoparticles. The thin films were analyzed by XRD, HRSEM, FT-IR spectroscopy, and also the UV-Vis that shows a transmittance up to $75 \%$.

\section{Experimental Methods}

This work relied on Zinc acetate (Merck), potassium hydroxide $\mathrm{KOH}$ (Baker), and methanol as the chemical reagents. In a typical procedure $0.8977 \mathrm{~g}$. of $\mathrm{KOH}$ was dissolved into $100 \mathrm{~mL}$ of methanol, and it was then heated at $60^{\circ} \mathrm{C}$. This mixture was then put under vigorous stirring for 30 minutes in order to obtain a homogenous solution Thereafter, a solution of $2.107 \mathrm{~g}$. of zinc acetate dihydrate was prepared in $60 \mathrm{~mL}$ of deionized water. Finally, the zinc solution was added to $\mathrm{KOH}$ solution and it was stirred vigorously at $60^{\circ} \mathrm{C}$ for 90 minutes in order to obtain a well-dissolved sol.

The solution obtained from this reaction was divided into three parts. The first part of the divided solution contains impurities, so it was characterized in order to have a reference of the properties of the $\mathrm{ZnO}$ particles. The second one obtained from the experiment was washed two times according to the modified Meulekamp's method [18]; this method was simplified because we use only methanol as the dispersing agent and a centrifuge to precipitate the nanoparticles to remove the byproducts as a difference from Meulekamp's where he uses alkanes to provoke the precipitation of the nanoparticles, and the redispersion was done with ethanol. In the third part, the product was washed three times, again with the same method described in this work. Figure 1 shows the flux diagram of the procedure for the synthesis of $\mathrm{ZnO}$ nanoparticles and the purifying process. In order to proceed with the characterization of the product, the solvent was removed at each step by heating it at $60^{\circ} \mathrm{C}$ until a white, dry powder, containing the $\mathrm{ZnO}$ nanoparticles, was obtained. The

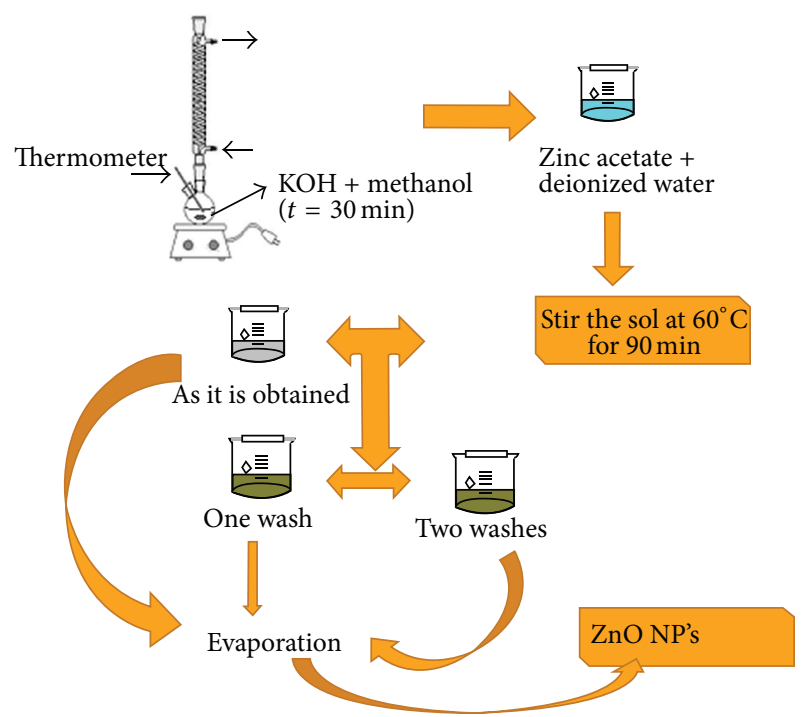

FIgURE 1: Flux diagram of the $\mathrm{ZnO}$ nanoparticles synthesis.

structure of the white $\mathrm{ZnO}$ nanocrystals obtained at each step was characterized by powder X-ray diffraction (XRD) using a D8 Discover diffractometer for crystal phase identification, and the average crystalline sizes were estimated using the Scherrer's formula. This work performed the washing and the redispersion method 3 times that was enough to reach pure $\mathrm{ZnO}$ nanoparticles. Also the nanoparticles obtained from each step were annealed at $600^{\circ} \mathrm{C}$ in order to remove the byproducts which could not be done with the process described before and analyze the effect of this temperature on the shape, size, and composition of the nanoparticles.

The Fourier transmission infrared (FT-IR) spectra of the powders in $\mathrm{KBr}$ were obtained using a Fourier transmission infrared spectrometer Bruker model vector 22. The morphology and shape of $\mathrm{ZnO}$ nanoparticles were studied with a conventional transmission electron microscopy, with a JEOL100C equipment.

So the thin films were deposited using a spin coater model CHEMAT with $2000 \mathrm{rpm}$ with spin-up time of 20 seconds; after depositing each layer, it was preheated at $60^{\circ} \mathrm{C}$ for 3 minutes in a conventional oven to remove the solvent. The layers deposited were characterized each five times until we deposit 30-layer film; the film after the finished layer deposition was annealed at $600^{\circ} \mathrm{C}$ just to increase the adherence to the substrate. The films obtained were analyzed with HRSEM, UV-Vis, XRD, and perfilometry in order to obtain the thickness.

\section{Results and Discussion}

\subsection{Characterization of NP's Powders}

3.1.1. Composition from NP's Powders. The Fourier transform infrared spectroscopy (FT-IR) shows the vibrational modes that correspond to the $\mathrm{ZnO}$ compound and also the byproducts that appeared due to the effect of the unreacted materials. Figures 2(a) and 2(b) show the FT-IR spectra of 


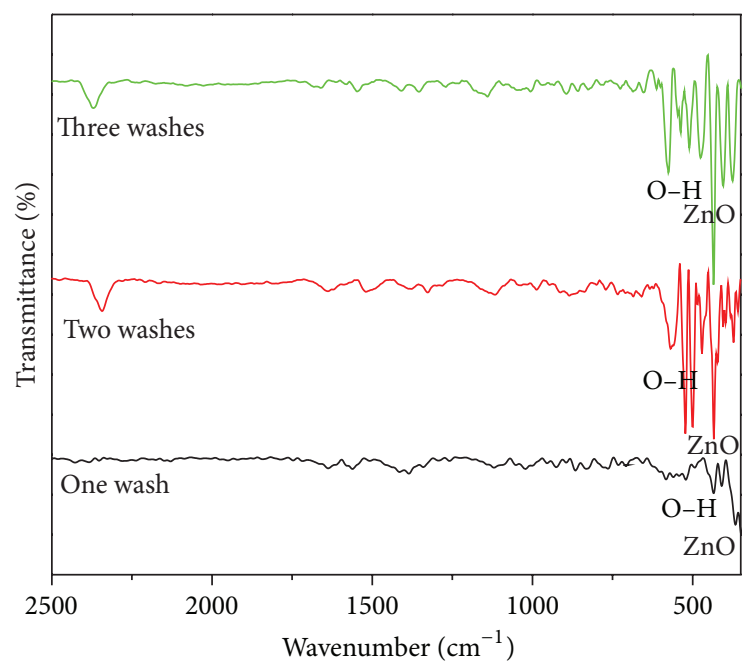

(a)

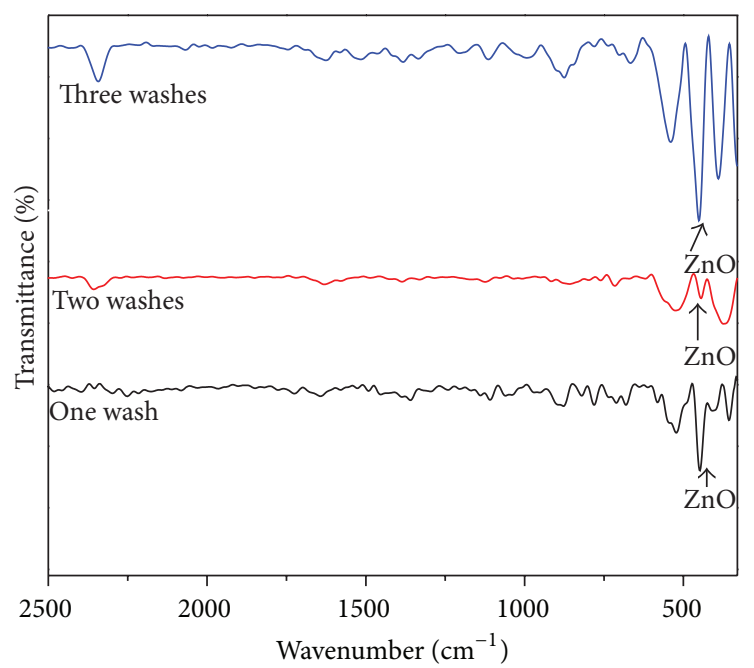

(b)

FIGURE 2: FT-IR spectra of $\mathrm{ZnO}$ powders (a) after one, two, and three times precipitation-redispersion method, (b) the same conditions after annealing process at $600^{\circ} \mathrm{C}$.

$\mathrm{ZnO}$ nanoparticles obtained during the first, second, and third steps and after an annealing treatment, respectively. The main absorption bands in the FT-IR spectra correspond to asymmetric and symmetric $\mathrm{C}=\mathrm{O}$ bands in 1380 and $1600 \mathrm{~cm}^{-1}$; the peak near $2300 \mathrm{~cm}^{-1}$ corresponds to $\mathrm{CO}_{2}$ vibrational modes; for this compound also characteristics modes of $\mathrm{O}-\mathrm{H}$ bending hydroxyl groups appear at $567 \mathrm{~cm}^{-1}$; finally in the range of $500-525 \mathrm{~cm}^{-1}$ and under $400 \mathrm{~cm}^{-1}$ the signals correspond to carboxyl acid groups. The $\mathrm{ZnO}$ stretching band of $\mathrm{ZnO}$ was located at $430 \mathrm{~cm}^{-1}$ [19]. After the annealing treatment, the FT-IR spectra show a decrease of intensity of the signals associated with byproducts, and a more definite and clear signal corresponding to $\mathrm{ZnO}$ can be observed. This is due to the evaporation of byproducts after the annealing treatment; also the $\mathrm{OH}$ compounds started to evaporate completely at $600^{\circ} \mathrm{C}$. This temperature was selected because almost all carbon compounds evaporate, as shown in the FTIR spectra Figure 2(a).

3.1.2. Structure from NP's Powders. Figure 3 shows the X-ray spectra of the $\mathrm{ZnO}$ powders as obtained, in the first, second, and third step (without any annealing process). The samples were polycrystalline in character, and the corresponding spectra fit well with hexagonal, wurtzite type $\mathrm{ZnO}$ from ASTM cards. The peaks corresponding to the (100), (002), (101), (102), (110), (103), (200), (112), and (201) planes were observed. According to these results, it can be seen that the purifying process increases the intensity of the signal associated with the (101) plane.

The average crystallite size of the nanoparticles was estimated from the XRD patterns using Scherrer's formula, $(D=$ $0.9 / \mathrm{FWHM} \cos \theta$ ), where $D$ is the average grain size, FWHM is the full, wide, at a hall maximum, and $\theta$ is the grades at $2 \theta$, yielding a value of $30 \mathrm{~nm}$ for the synthesized nanoparticles.

Figure 3 inset shows the X-ray pattern of the $\mathrm{ZnO}$ nanoparticles after an annealing process where it is clearly seen that the size and form of the nanoparticles are not modified after this process. That means that there is not a recrystallization process that can contribute to the growth of the nanoparticles after the synthesis.

3.1.3. Morphology of the NP's Powders. Figure 4 shows the micrographs of $\mathrm{ZnO}$ nanoparticles right after preparation and after washing in methanol, as observed by conventional TEM. The structure of the nanopowders synthesized corresponds to the wurtzite (hexagonal form) which confirms those results obtained from X-ray diffraction and Figure 4(a) inset micrograph, and it can be observed that the narrowing in crystallite size distribution and stabilization depend on the washing process, as seen in Figures 4(a), 4(b), 4(c), and 4(d). In fact, from the samples obtained in the first washing, in Figure 4(b) it can be noted that the agglomeration of particles quickly takes place; this is also observed in micrograph (a). These effects are explained from the results obtained from transmittance spectra and are due the great quantity of byproducts present in the solution. On the other hand, the size dispersion is not homogeneous; when the washing process continues the size starts to be homogeneous as it is shown in Figure 4(d). This behavior can be due to a decrease in the by-product concentration as the washing process steps increase, and this provokes the reduction of the kinetic energy around the nanoparticles with the byproducts.

3.1.4. Colloid Stability. After 24 hours of the first step, the $\mathrm{ZnO}$ nanoparticles dissolved in methanol showing a precipitation in the bottom of the test probe. It is usual to describe this behavior by saying that the $\mathrm{ZnO}$ nanoparticle does not show stability in methanol. However, it is observed that after washing it in Meulekamp's (modified) method, the stability of the colloidal solution increased. That is, the time required in order for the particles to precipitate in the bottom of the test probe increased up to 12 days for samples washed three times. 


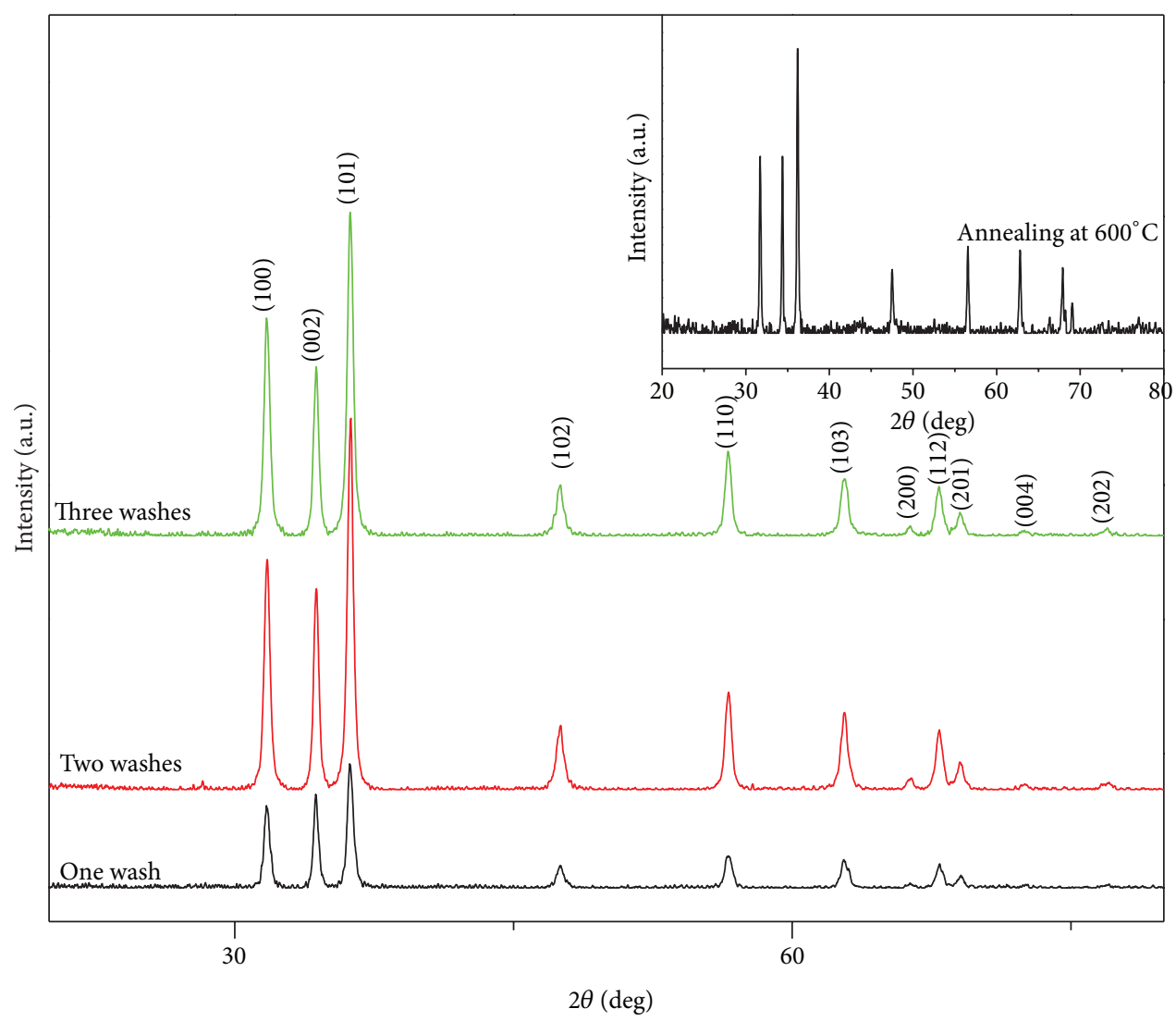

FIGURE 3: $\mathrm{XRD}$ diffractograms of $\mathrm{ZnO}$ nanoparticles of size $30 \mathrm{~nm}$, washed a one, $\mathrm{b}$ two, and c three times, and the inset graph is the $\mathrm{X}$-ray pattern with an annealing process done at $600^{\circ} \mathrm{C}$ of the powder samples.

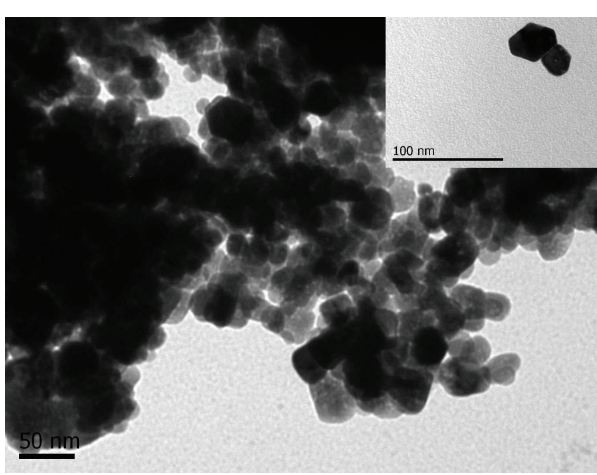

(a)

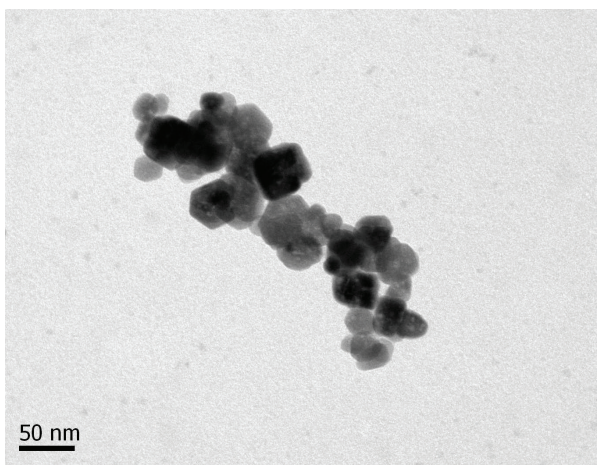

(c)

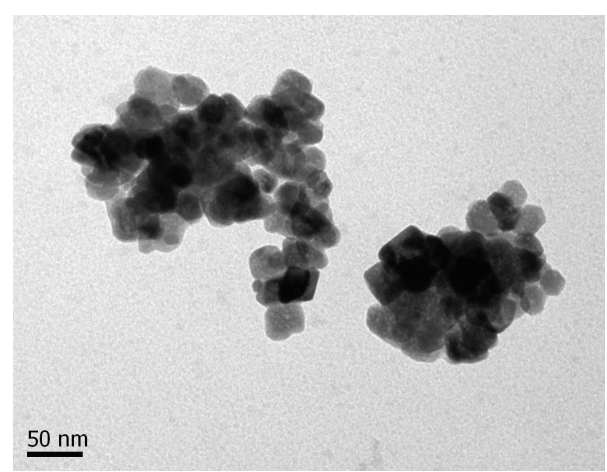

(b)

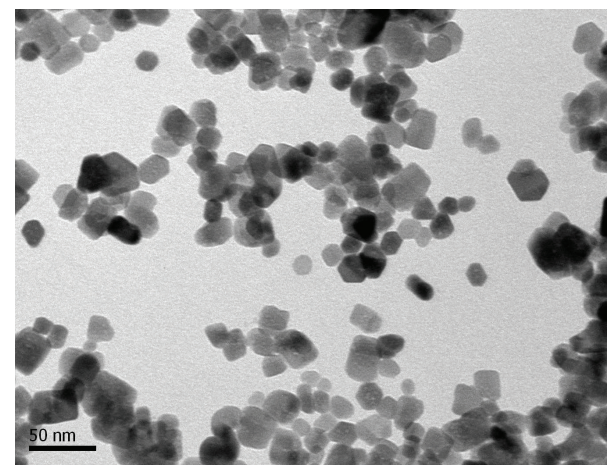

(d)

FIGURE 4: TEM micrographs of colloidal $\mathrm{ZnO}$ nanoparticles. (a) As it was obtained, (b) one, (c) two, and (d) three washes of $\mathrm{ZnO}$ nanoparticles. 


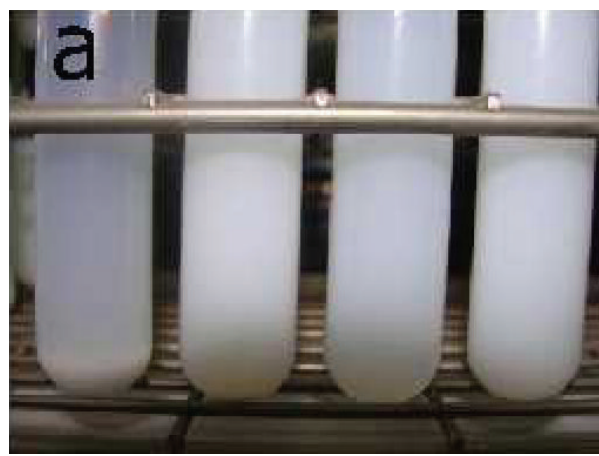

(a)

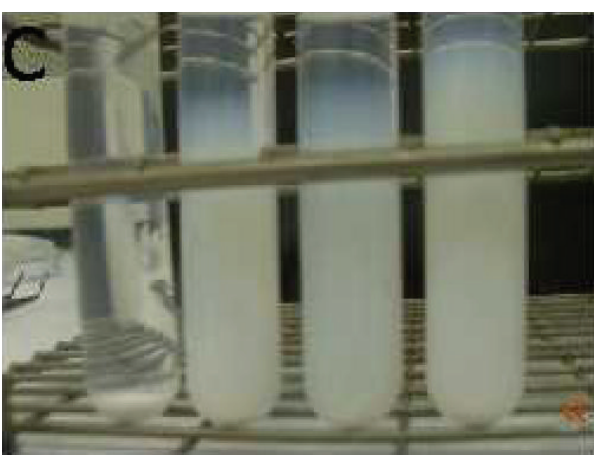

(c)

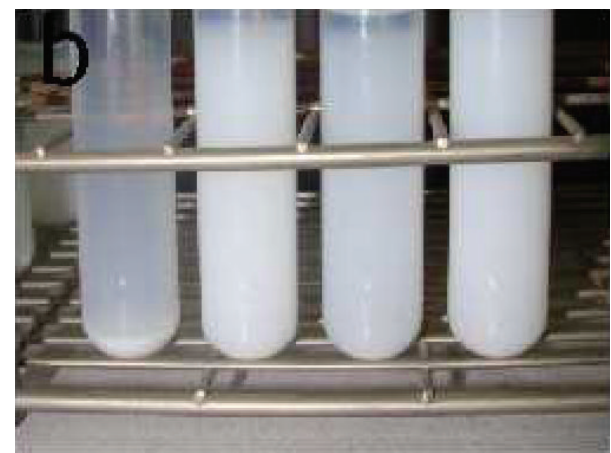

(b)

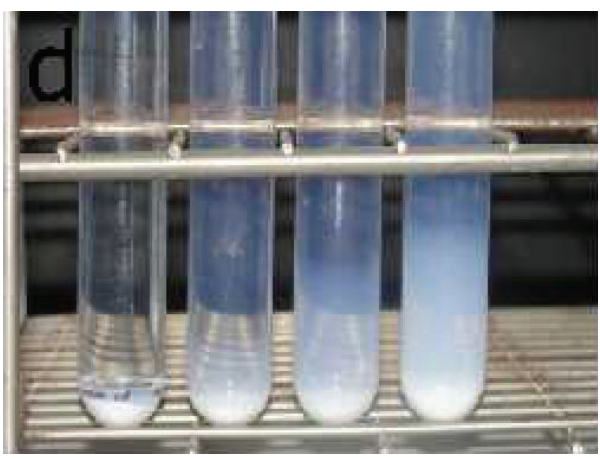

(d)

Figure 5: Pictures of the colloidal solution (a) first day, (b) second day, (c) 12 days, and (d) 24 days (the essay tubes from the left side start the sol as it is obtained, one wash, two washes, and finally three washes).

Based on these results, it can be concluded that the decrease in the byproducts, through the washing steps, increased the stability of the $\mathrm{ZnO}$ nanoparticles in methanol according to TEM micrographs. In fact in the literature many authors say that stability depends on the amount of acetate groups bound to the surface of $\mathrm{ZnO}$ nanoparticles, and that most of the byproducts were removed from the solution according of the FTIR spectra performed in this work.

Figure 5(a) shows the picture of $\mathrm{ZnO}$ nanoparticles dissolved in methanol and the corresponding precipitation observed right after preparation and after the first, second, and third washing steps. Similar pictures were taken on day one (Figure 5(a)), day two (Figure 5(b)), day 12 (Figure 5(c)), and day 24 (Figure 5(d)) in order to check on the stability of the $\mathrm{ZnO}$ nanoparticles.

Homogeneous dispersion of $\mathrm{ZnO}$ nanoparticles was maintained for samples washed three times and after 24 days. Nevertheless, precipitation of nanoparticles increased with time. Finally, interactions of NP's to NP's lessened when there was no byproducts, such as the acetate group that has a very high electronegativity which provokes agglomeration of components.

3.2. Deposition of the Spin Coated Films. The thin films were deposited using a spin coater CHEMAT model KW-4A with 20 seconds of spin-up at 2000 RPM, and after each deposit the layer was preheated at $60^{\circ} \mathrm{C}$ just to remove the solvent; this condition was maintained until reaching a specific number of layers in order to characterize and analyze the evolution of the way nanocrystals deposit onto a substrate. But we observe that the resultant films do not have good adherence to the substrate so we have to make a post annealing process for this film at $600^{\circ} \mathrm{C}$. This temperature is well studied and has the best result and increases the adherence of the coated film to the substrate.

3.2.1. Structure of the Thin Films. After the first layer of $\mathrm{ZnO}$ sol film was coated and preheated, the nanocrystals were deposited gradually onto the substrate. Since the glass substrate is an amorphous material, the nanoparticles should be randomly oriented; correspondingly, the crystals were also randomly oriented. However, the (lllll $\left.\begin{array}{lll}0 & 2\end{array}\right)$ plane of $\mathrm{ZnO}$ has the minimum surface free energy [20], so most of the crystals deposit preferentially along the $\left(\begin{array}{ll}0 & 0\end{array}\right)$ direction (namely, the $c$-axis direction), and only a small part of the crystals deposits along other directions. After the second layer of $\mathrm{ZnO}$ sol film was coated and preheated and the new crystals were deposited using the former layer as a growth template; therefore, some new crystals still deposit along other directions rather than the $\left(\begin{array}{lll}0 & 0 & 2\end{array}\right)$ direction. This can explain why samples $\mathrm{A}$ and $\mathrm{B}$ have the $\left(\begin{array}{lll}1 & 0 & 0\end{array}\right)$ and $\left(\begin{array}{lll}1 & 0 & 1\end{array}\right)$ peaks besides the $\left(\begin{array}{lll}0 & 0 & 2\end{array}\right)$ peak as shown in Figure 6. However, with the increase of the film thickness, the grains begin to coalesce and grow in a direction to minimize the energy of the substrate-film interface and free surface energy [21]. In the single-layer crystal growth process, the interaction between $\mathrm{ZnO}$ crystals played a dominant role in the deposition orientation. Because the crystals along the $\left(\begin{array}{lll}0 & 0 & 2\end{array}\right)$ direction deposit easily and 


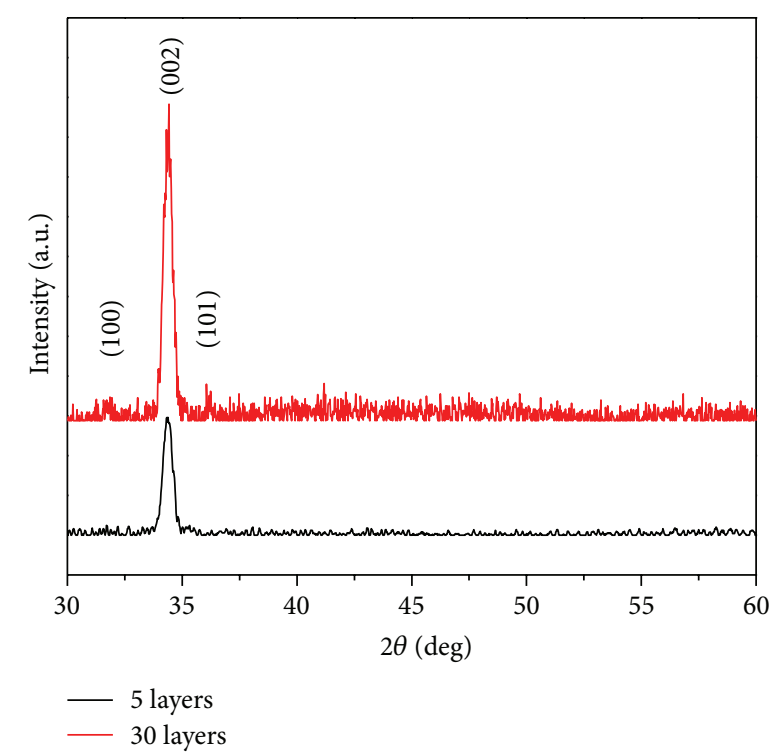

Figure 6: $X$ ray pattern for deposited thin films 5 and 30 layers on glass substrate with highly $c$-axis orientation.

faster, those crystals depositing along other directions are soon suppressed [22]. When the layer crystals were formed, they were nearly all oriented along the $\left(\begin{array}{lll}0 & 0 & 2\end{array}\right)$ direction. After the total layers were coated, the $\mathrm{ZnO}$ thin film was postannealed. In the annealing process, the diffusion and migration of atoms between neighboring layers happened to make coalescence between the neighboring nanoparticles. Finally, the nanoparticles through the entire film thickness were deposited. The $\mathrm{ZnO}$ thin film deposited by sol-gel method is a multilayered system. However, when the film was given a suitable annealing treatment including appropriate annealing temperature and time, the resulting $\mathrm{ZnO}$ thin film has an interface between neighboring nanoparticles and in fact formed an entire nanoparticles layer, which has been observed in this work. It should be pointed out that a suitable annealing treatment is very important for the formation of $\mathrm{ZnO}$ nanoparticles coalescence with columnar structure by colloidal solution. For example, Zhu et al. [23] found that the columnar structure can be formed only after a certain incubation time from a film that featured a granular grain structure. The colloidal technique is a wet-chemical method. The film deposition process by the colloidal method is very different from those by other physical techniques such as pulsed laser deposition, magnetron sputtering, and electron beam evaporation. Accordingly, the total growth process of $\mathrm{ZnO}$ thin films including nucleation, crystal growth, and coarsening is also very different from colloidal solution method and other physical deposition methods because in this case we have a solution with formed well-defined nanoparticles to hexagonal form. The studies on the deposit mechanisms of $\mathrm{ZnO}$ thin films prepared by colloidal solution method are very important for getting high-quality films applied to optoelectronic devices or as a gas sensor.

3.2.2. Morphology of Thin Films. So far, many research groups have prepared highly $c$-axis oriented $\mathrm{ZnO}$ thin films by sol-gel method [24]. Those $\mathrm{ZnO}$ thin films are preferentially oriented along the $c$-axis and are connected with the nature of $\mathrm{ZnO}$ as well as film preparation conditions. Owing to the minimum surface free energy of the $\left(\begin{array}{lll}0 & 0 & 2\end{array}\right)$ plane [23], the (llll $\left.0 \begin{array}{ll}0 & 2\end{array}\right)$ direction (namely, $c$-axis orientation) of a $\mathrm{ZnO}$ thin film is the most thermodynamically favorable growth direction. This is the intrinsic factor leading to $\mathrm{ZnO}$ crystals preferentially growing along the $c$-axis orientation. For example, Ohyama et al. [25] and Fujimura et al. [20] prepared highly $c$-axis oriented $\mathrm{ZnO}$ thin films on glass substrates by sol-gel method and pulsed laser deposition, respectively. Since the glass is an amorphous material, there is no epitaxial relationship between $\mathrm{ZnO}$ and the substrate. However, these $\mathrm{ZnO}$ thin films are not randomly oriented. Furthermore, Dong et al. [26] found that even if the $\mathrm{ZnO}$ thin film is as thin as $15 \mathrm{~nm}$, it is still oriented along the $c$ axis. These results indicate that the intrinsic factor plays a very important role for $\mathrm{ZnO}$ crystals to be preferentially oriented along the $c$-axis. On the other hand, the film preparation conditions are the extrinsic factors affecting the growth orientation of $\mathrm{ZnO}$ thin films. For example, for the $\mathrm{ZnO}$ thin films deposited by sol-gel method, the sol stabilizer has an important effect on crystal orientation. But in this case the nanoparticles are formed so it is only the way that they are deposited, and $c$-axis oriented $\mathrm{ZnO}$ thin films deposited by this method have been proposed up to now. For example, Wang et al. [27] think that the growth of highly $c$-axis oriented $\mathrm{ZnO}$ thin films is a self-assembly process in which a dipoledipole interaction between the polar nanograins plays a great role in the crystal growth. A sketch of the $\mathrm{ZnO}$ thin film selfassembly mechanism along the $c$-axis. For another example, Zhu et al. [23] propose that the growth of highly $c$-axis oriented $\mathrm{ZnO}$ thin film is a self-buffer layer process in which every layer will act as a buffer layer for next one, promoting a heterogeneous nucleation process. The authors think that the deposition process of highly $c$-axis oriented $\mathrm{ZnO}$ thin films deposited by a spin coating method is not only a self-assembly process but also a self-template process. A schematic sketch of the growth mechanism of self-template process is shown this is according to the HRSEM micrographs Figures 7(a) and 7(b) corresponding to 5 layer deposition to 30 layer deposition that show this kind of process.

According to the HRSEM micrographs we propose the mechanism of deposition for these nanoparticles onto glass substrate. The Figure 8 shows in general the way that the nanoparticles are dispersed when they are spin up onto the substrate; the nanoparticles are dispersed at nonuniform way but as the number of layers increases the nanoparticles cover gradually the substrate but those ones do not have good adherence until the resultant coated films are postannealed. This process provokes the coalescence between them, and the adherence increases with this process. It corresponds directly with the transmittance spectra which shows a characteristic shoulder at 300 to $350 \mathrm{~nm}$; this is referred to the porosity that exists on the substrate.

3.2.3. Optical Properties of Thin Films. Figure 9 presents the optical transmittance spectra of $\mathrm{ZnO}$ thin films with different thickness and deposit layers. It is clear that all the 


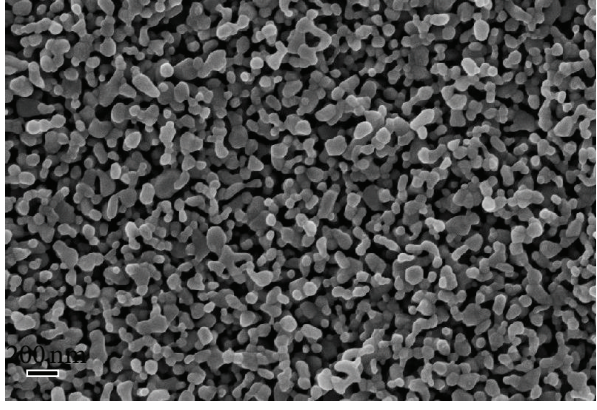

(a)

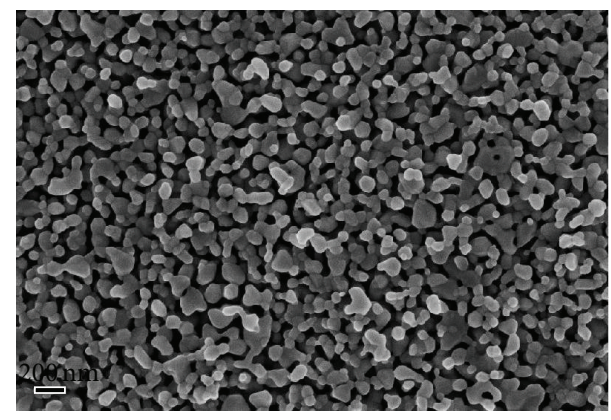

(b)

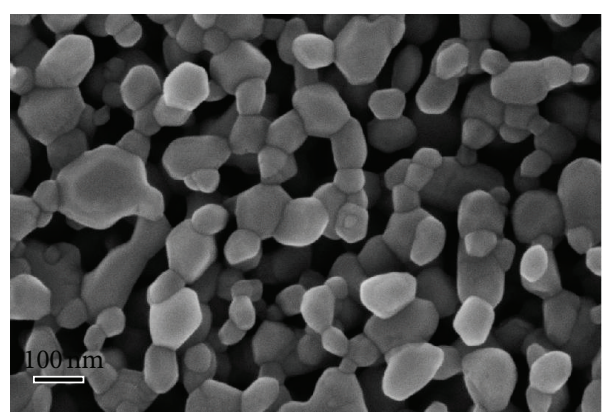

(c)

FIGURE 7: HRSEM micrographs for (a) 5, (b) 30 layers, and (c) showing the coarsening after annealing process.
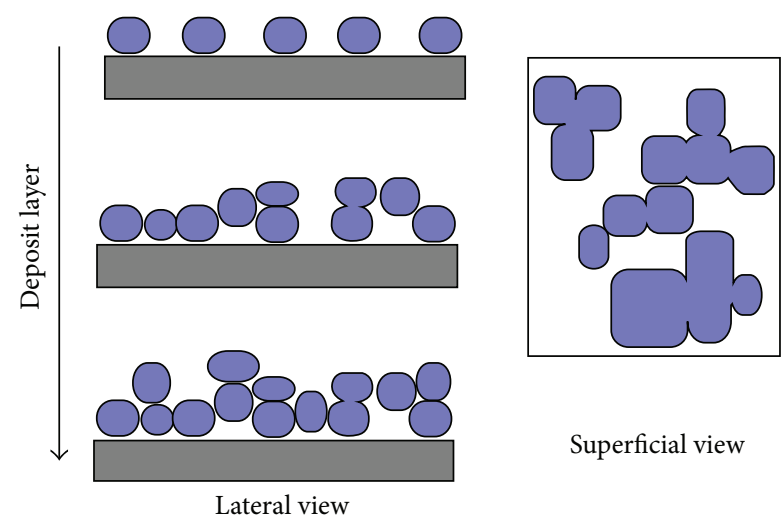

Superficial view

FIGURE 8: A proposed mode of deposit of the colloidal nanoparticlesand it's coalescence.

samples have high transmittance in the visible range, and the average is about $75 \%$. Therefore, within the ranges from 200 to $360 \mathrm{~nm}$, film thickness has almost no effect on the transmittance it corresponds to 5 layers to 15 layer film. However, compared with samples from 5, 10, 15, and 20 layers, the samples with 25 and 30 layers have a more uniform transmittance in the whole visible range $(400-700 \mathrm{~nm})$. This may be due to the decreased optical scattering caused by the decrease of grain boundary density owing to the increase of grain size. Also the shoulder starts to decrease; this is due of the densification of the nanoparticles as film. From the above results, it can be known that $\mathrm{ZnO}$ thin films prepared by the colloidal method have high transmittance in the visible range, which can be used as transparent window materials

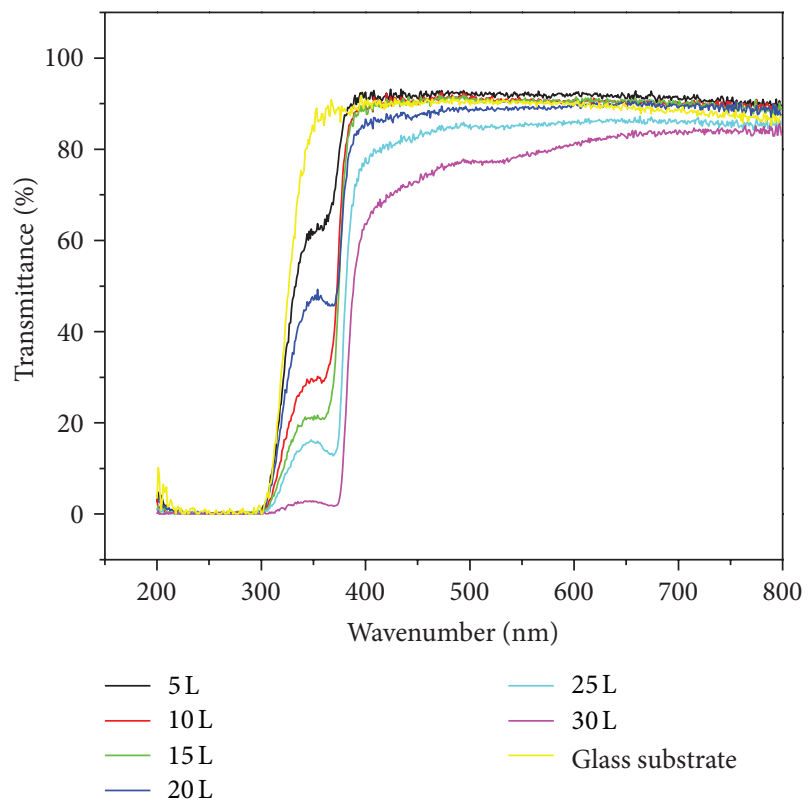

FIGURE 9: Optical transmittance up to $75 \%$ for spin coated films, for 5 to 30 layers.

in many optoelectronic devices [28]. Furthermore, it should be mentioned that Sharma and Mehra [21] found that the transmittance of $\mathrm{Co}$ and $\mathrm{Al}$ codoped $\mathrm{ZnO}$ thin films prepared by the sol-gel method decreased in the visible range when the film thickness was more than $200 \mathrm{~nm}$. They thought that the increase of scattering owing to the large roughness in thicker 


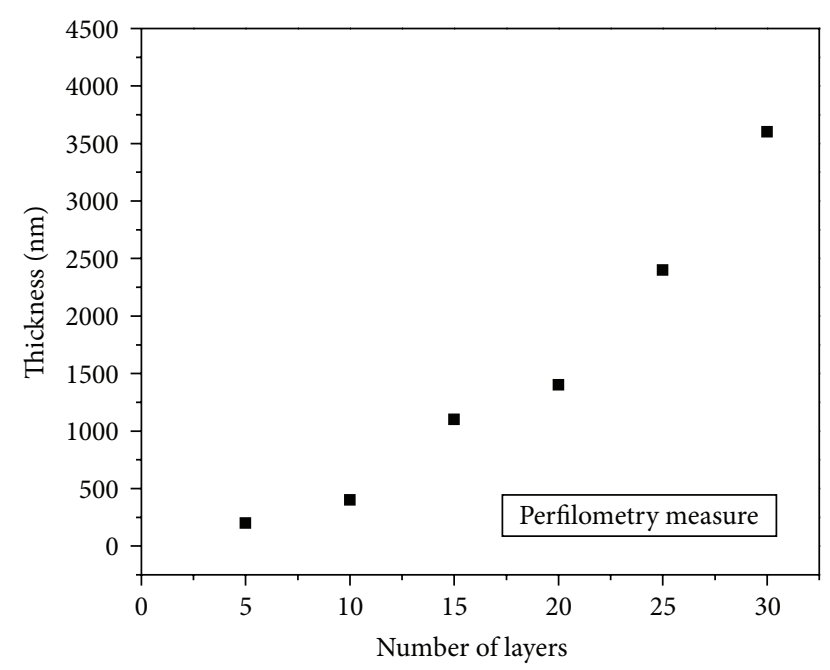

FIGURE 10: Thickness of films obtained by perfilometry according to the number of layers.

films and oxygen vacancies resulted in a decrease in optical transmittance.

So the thickness of the films obtained in this work was analyzed using perfilometry (see Figure 10) where we clearly saw that the minimum thickness obtained depositing a film of 5 layers corresponds to $200 \mathrm{~nm}$, and the maximum thickness of depositing 30 layers reaches $3600 \mathrm{~nm}$. It is easy to say that this technique to deposit colloidal $\mathrm{ZnO}$ nanoparticles films has no homogeneous dispersion but is well defined to use this as a gas sensor because there is a lot of contact surface along to the substrate as the coalescence that permits the interaction between each particle deposited as it is observed in Figure 7.

\section{Conclusions}

$\mathrm{ZnO}$ nanoparticles were synthesized by solution method starting by zinc acetate dihydrate and $\mathrm{KOH}$. In order to remove byproducts, the resulting powders were washed one, two, and three times with modified Meulekamp's method. FTIR measurements show that the washing process is effective in removing byproducts containing $\mathrm{OH}$ radicals and acetates, among other compounds as a definite signal corresponding only to $\mathrm{ZnO}$, which was finally obtained. The powders were polycrystalline with a hexagonal, wurtzite-type $\mathrm{ZnO}$ structure. The average crystallite size of $\mathrm{ZnO}$ nanoparticles was in the order of $25 \mathrm{~nm}$, and no significant change in the size of the nanoparticles was observed even after the annealing process. Evolution of the stability of $\mathrm{ZnO}$ nanoparticles dissolved in methanol was observed as a function of the number of washing steps finaly that three times is enough to purify these nanoparticles obtained from this method.

Rapid precipitation of $\mathrm{ZnO}$ nanoparticles was observed immediately for samples with no washing process, while in the case of solutions washed one, two and three times, the stability increased up to 12 days. However, as time elapsed the homogeneity was not stable, and rather precipitation was observed even for samples washed 3 times. The effect of washing of $\mathrm{ZnO}$ powders play a key role in the stability of
$\mathrm{ZnO}$ nanoparticles dissolved in methanol. As in some applications, stability is relevant, it is necessary a detailed study on the deposition variables in solution method in order to avoid early precipitation of $\mathrm{ZnO}$ nanoparticles. This study is in due course.

Finally, this work is remarkable because we discovered that the stability of the nanoparticles could be achieved without the interaction with surfactants any solvents as hexane, heptane, and so forth, and this stability depends directly on the washing step process.

According to the deposited films from colloidal solution the optical transmittance is up to $75 \%$ and the deposited nanoparticles are in preferential orientation in 002 plane called the $c$-axis; this due the lower energy that this plane has. So before the annealing process there is no adherence to the substrate, but when it is realized the nanoparticles start to coalesce, increasing the adherence to the substrate.

\section{Acknowledgments}

The authors thank Mrs. Lourdes Rojas from Laboratorios Centrales (CINVESTAV-IPN) for TEM characterization of $\mathrm{ZnO}$ samples. This work was supported by Consejo Nacional de Ciencia y Tecnologia (CONACyT) through Project no. 166601 .

\section{References}

[1] A. B. Djuriić, A. M. C. Ng, and X. Y. Chen, "ZnO nanostructures for optoelectronics: material properties and device applications," Progress in Quantum Electronics, vol. 34, no. 4, pp. 191259, 2010.

[2] S. J. Pearton, D. P. Norton, K. Ip, Y. W. Heo, and T. Steiner, "Recent progress in processing and properties of $\mathrm{ZnO}$," Progress in Materials Science, vol. 50, no. 3, pp. 293-340, 2005.

[3] G. C. Yi, C. Wang, and W. I. Park, "ZnO nanorods: synthesis, characterization and applications," Semiconductor Science and Technology, vol. 20, no. 4, article S22, 2005.

[4] S. Y. Lee, E. S. Shim, H. S. Kang, S. S. Pang, and J. S. Kang, "Fabrication of $\mathrm{ZnO}$ thin film diode using laser annealing," Thin Solid Films, vol. 473, no. 1, pp. 31-34, 2005.

[5] Y. J. Zeng, Z. Z. Ye, W. Z. Xu et al., "Realization of p-type ZnO films via monodoping of Li acceptor," Journal of Crystal Growth, vol. 283, no. 1-2, pp. 180-184, 2005.

[6] H. S. Kang, B. D. Ahn, J. H. Kim et al., "Structural, electrical, and optical properties of $p$-type $\mathrm{ZnO}$ thin films with Ag dopant," Applied Physics Letters, vol. 88, no. 20, Article ID 202108, 2006.

[7] B. Yao, D. Z. Shen, Z. Z. Zhang et al., Journal of AppliedPhysics, vol. 99, Article ID 12351, 2006.

[8] Y. R. Jang, K. Yoo, and S. M. Park, "Properties of ZnO thin films grown on $\mathrm{Si}$ (100) substrates by pulsed laser deposition," Journal of Materials Science and Technology, vol. 26, no. 11, pp. 973-976, 2010.

[9] S. Komarneni, M. Bruno, and E. Mariani, "Synthesis of $\mathrm{ZnO}$ with and without microwaves," Materials Research Bulletin, vol. 35, no. 11, pp. 1843-1847, 2000.

[10] C. H. Lu and C. H. Yeh, "Influence of hydrothermal conditions on the morphology and particle size of zinc oxide powder," Ceramics International, vol. 26, no. 4, pp. 351-257, 2000. 
[11] Z. Zhou, H. Deng, J. Yi, and S. Liu, "A new method for preparation of zinc oxide whiskers," Materials Research Bulletin, vol. 34, no. 10-11, pp. 1563-1567, 1999.

[12] C. H. Lu and C. H. Yeh, "Emulsion precipitation of submicron zinc oxide powder," Materials Letters, vol. 33, no. 3-4, pp. 129132, 1997.

[13] U. Pal, J. G. Serrano, P. Santiago, G. Xiong, K. B. Ucer, and R. T. Williams, "Synthesis and optical properties of $\mathrm{ZnO}$ nanostructures with different morphologies," Optical Materials, vol. 29, no. 1, pp. 65-69, 2006.

[14] W. Bai, K. Yu, Q. Zhang et al., "Large-scale synthesis of zinc oxide rose-like structures and their optical properties," Physica E, vol. 40, no. 4, pp. 822-827, 2008.

[15] R. Wahab, S. G. Ansari, Y. S. Kim, M. Song, and H. Shin, "The role of $\mathrm{pH}$ variation on the growth of zinc oxide nanostructures," Applied Surface Science, vol. 255, no. 9, pp. 4891-4896, 2009.

[16] A. P. A. Oliveira, J. Hochepied, F. Grillon, and M. Berger, "Controlled precipitation of zinc oxide particles at room temperature," Chemistry of Materials, vol. 15, no. 16, pp. 3202-3207, 2003.

[17] Q. P. Zhong and P. Matijevic, "Preparation of uniform zinc oxide colloids by controlled double-jet precipitation," Journal of Materials Chemistry, vol. 6, pp. 443-447, 1996.

[18] E. A. Meulenkamp, "Synthesis and growth of $\mathrm{ZnO}$ nanoparticles," The Journal of Physical Chemistry B, vol. 102, pp. 5566$5572,1998$.

[19] S. Suwanboon, "Structural and optical properties of nanocrystalline $\mathrm{ZnO}$ powder from sol-gel method," ScienceAsia, vol. 34, no. 1, pp. 31-34, 2008.

[20] N. Fujimura, T. Nishihara, S. Goto, J. Xu, and T. Ito, "Control of preferred orientation for $\mathrm{ZnOx}$ films: control of self-texture," Journal of Crystal Growth, vol. 130, no. 1-2, pp. 269-279, 1993.

[21] M. Sharma and R. M. Mehra, "Effect of thickness on structural, electrical, optical and magnetic properties of $\mathrm{Co}$ and $\mathrm{Al}$ doped $\mathrm{ZnO}$ films deposited by sol-gel route," Applied Surface Science, vol. 255, pp. 2527-2532, 2008.

[22] S. Nicolay, S. Fay, and C. Ballif, "Growth model of MOCVD polycrystalline ZnO," Crystal Growth and Design, vol. 9, no. 11, pp. 4957-4962, 2009.

[23] M. W. Zhu, J. H. Xia, R. J. Hong et al., "Heat-activated structural evolution of sol-gel-derived $\mathrm{ZnO}$ thin films," Journal of Crystal Growth, vol. 310, no. 4, pp. 816-823, 2008.

[24] P. T. Hsieh, Y. C. Chen, M. S. Lee, K. S. Kao, M. C. Kao, and M. P. Houng, "The effects of oxygen concentration on ultraviolet luminescence of $\mathrm{ZnO}$ films by sol-gel technology and annealing," Journal of Sol-Gel Science and Technology, vol. 47, no. 1, pp. 1-6, 2008.

[25] M. Ohyama, H. Kozuka, and T. Yoko, "Sol-gel preparation of $\mathrm{ZnO}$ films with extremely preferred orientation along (002) plane from zinc acetate solution," Thin Solid Films, vol. 306, no. 1, pp. 78-85, 1997.

[26] B. Z. Dong, G. J. Fang, J. F. Wang, W. J. Guan, and X. Z. Zhao, "Effect of thickness on structural, electrical, and optical properties of $\mathrm{ZnO}$ : Al films deposited by pulsed laser deposition," Journal of Applied Physics, vol. 101, Article ID 033713, 2007.

[27] J. Wang, Y. Qi, Z. Zhi, J. Guo, M. Li, and Y. Zhang, "A self-assembly mechanism for sol-gel derived $\mathrm{ZnO}$ thin films," Smart Materials and Structures, vol. 16, no. 6, p. 2673, 2007.

[28] J. C. Lee, K. H. Kang, S. K. Kim, K. H. Yoon, I. J. Park, and J. Song, "RF sputter deposition of the high-quality intrinsic and n-type $\mathrm{ZnO}$ window layers for $\mathrm{Cu}(\mathrm{In}, \mathrm{Ga}) \mathrm{Se}_{2}$-based solar cell applications," Solar Energy Materials and Solar Cells, vol. 64, no. 2, pp. 185-195, 2000. 

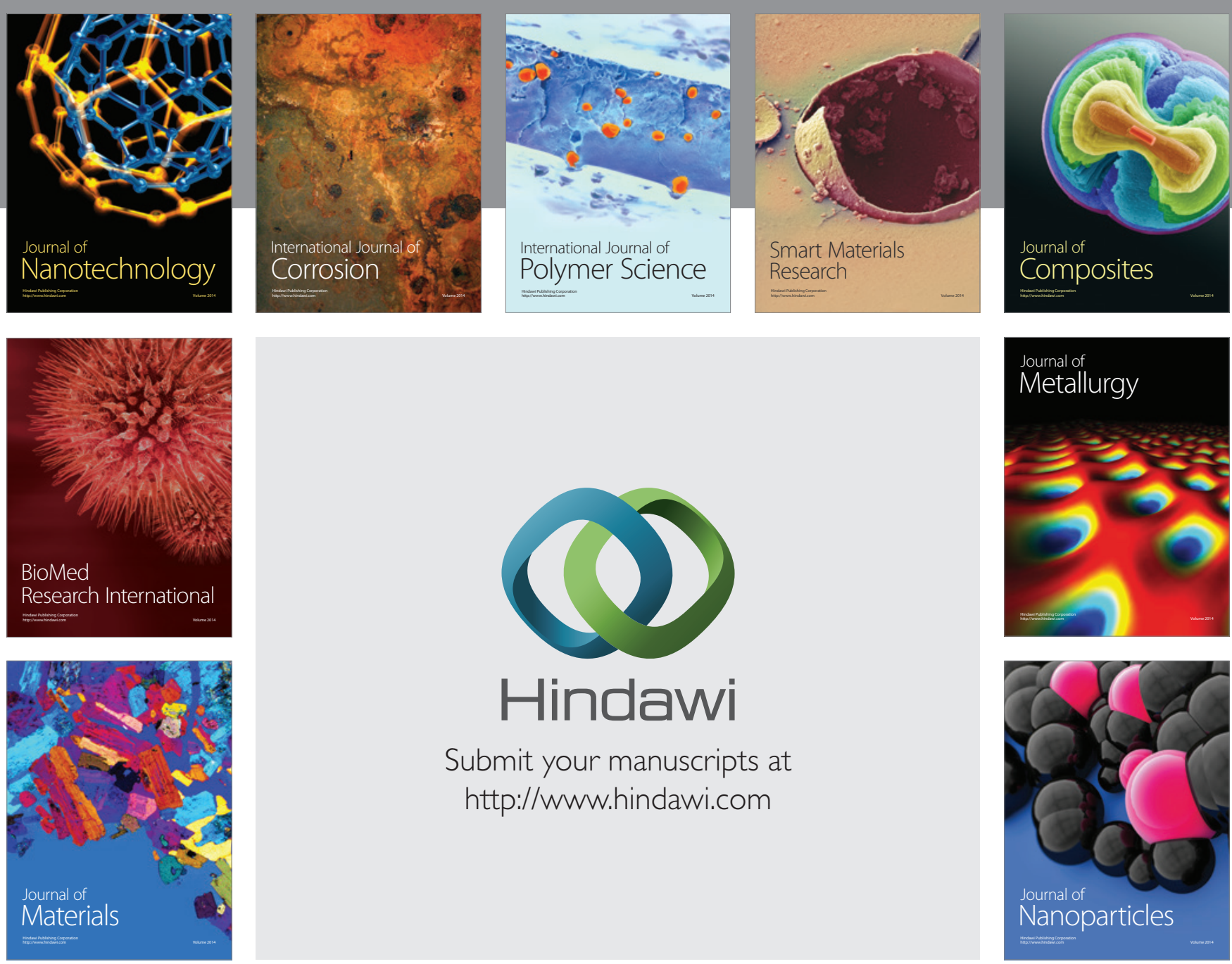

Submit your manuscripts at http://www.hindawi.com
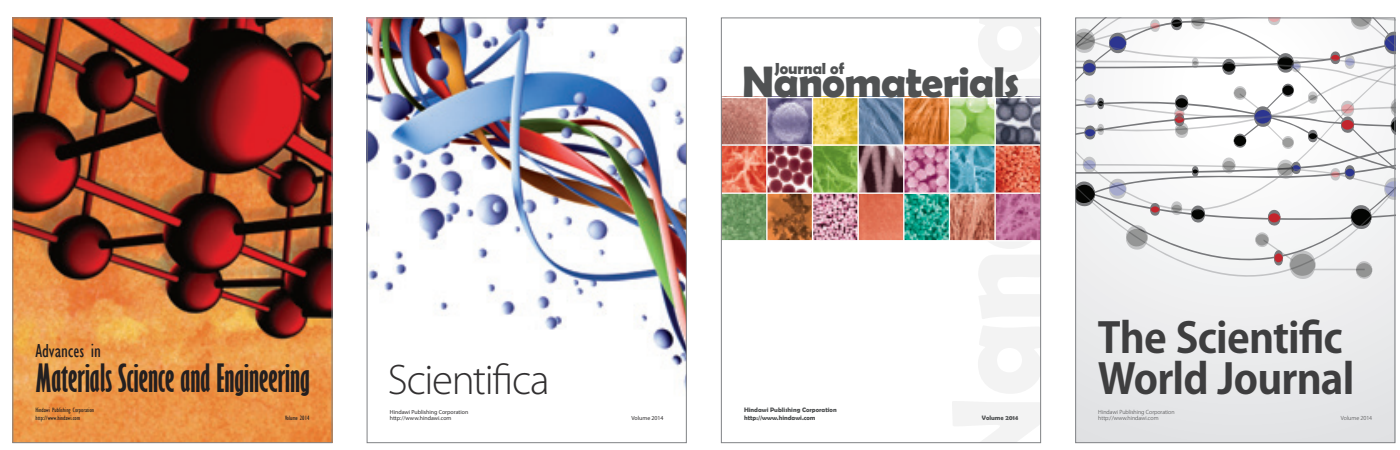

\section{The Scientific World Journal}
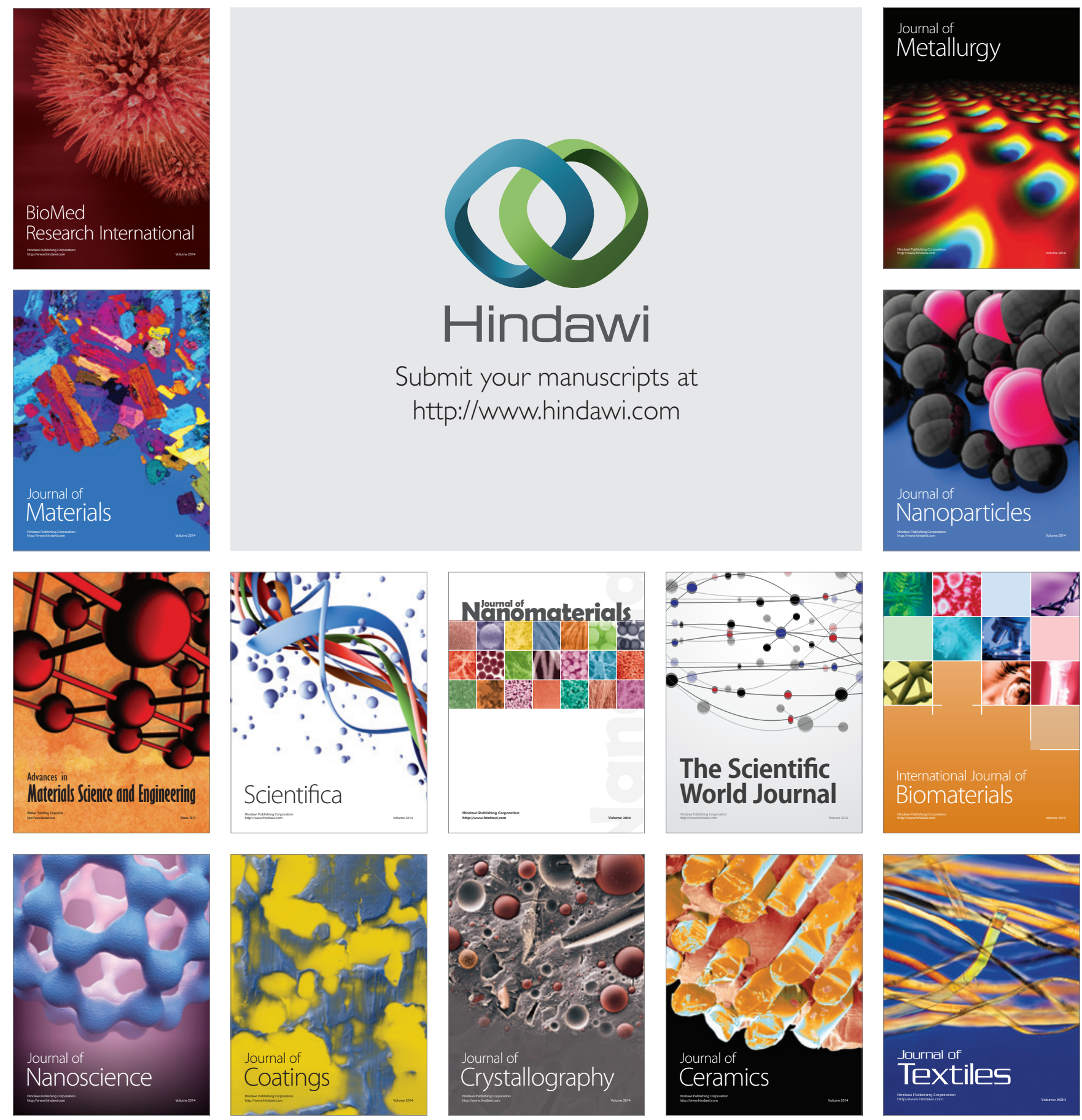